\title{
Funções de pedotransferência para as curvas de retenção de água e de resistência do solo à penetração em sistemas de manejo com plantas de cobertura permanente em citros
}

\author{
Pedotransfer functions for the soil water retention and soil resistance to penetration under \\ groundcover management systems in citrus
}

\author{
Jonez Fidalski ${ }^{\text {I }}$ Cássio Antonio Tormena ${ }^{\text {II }}$
}

\section{RESUMO}

A qualidade do solo em sistemas de manejo pode ser avaliada por indicadores simples ou por equações de pedotransferências. O objetivo deste estudo foi obter funções de pedotransferência (FPT) para as curvas de retenção de água (CRA) e de resistência do solo à penetração (CRP), em sistemas de manejo com plantas de cobertura permanente nas entrelinhas de laranjeira "Pêra". Avaliaram-se três tratamentos: gramínea Paspalum notatum roçada, leguminosa Arachis pintoi e vegetação espontânea com herbicida, em um Argissolo Vermelho distrófico latossólico, no noroeste do Paraná. Em maio de 2003, foram coletadas 216 amostras indeformadas de solo sob o rodado e o entrerrodado a 0,05-0,10m de profundidade. Avaliaram-se a densidade do solo (Ds), o teor de carbono orgânico (CO), o teor de água e a resistência do solo à penetração (RP) para ajustar as curvas de CRA e CRS em diferentes potenciais mátricos. As FPT foram dependentes da Ds, CO e tratamento para o ajuste das CRA $\left(R^{2}=0,94\right) e$ CRS $\left(R^{2}=0,87\right)$. O manejo da vegetação espontânea com herbicida aumenta a compactação do solo, reduz a retenção de água e aumenta a RP. A leguminosa reduz a retenção de água do solo e aumenta a RP, e a gramínea melhora a qualidade física e hídrica do solo nas entrelinhas. Alternativamente, as FPT exigem apenas as determinações dos teores de água e Ds para as CRA e CRS.

Palavras-chave: carbono orgânico, densidade do solo, manejo do solo, qualidade do solo.

\section{ABSTRACT}

The soil quality in management systems can be assessed by simple indicators or pedrotransfer functions. This study was aimed at obtaining pedotransfer function (PTF's) such as the soil water retention curve (SWRC) and the soil resistance to penetration curve (SRPC), under interrows groundcover management systems of the 'Pêra' orange. Three treatments were evaluated: bahiagrass mowed Paspalum notatum, perennial peanut Arachis pintoi and spontaneous vegetation with herbicide, in a Typic Paleudult, northwestern Paraná, Brazil. In May 2003, two hundred and sixteen undisturbed soil samples were collected under beneath and between the whell tracks at 0.05-0.10m depth. Soil bulk density $\left(D_{b}\right)$, organic carbon (OC), water content and soil resistance to penetration $(P R)$ were determined to fit the SWRC and SRPC in differents water potential. The $D_{b}$ and OC contributed to fit the SWRC and SRPC under groundcover management systems in the interrows. PTF's were depended $D_{b}$ OC and treatment to fit SWRC $\left(R^{2}=0.94\right)$ and SRPC $\left(R^{2}=0.87\right)$. Management on spontaneous vegetation using herbicide enhance soil compaction, decrease water retention and increase $P R$. Perennial peanut decrease soil water retention and increase $P R$, and bahiagrass groundcover enhance the soil physical and hydarulic quality in the interrows. Alternatively, PTF's required only to the determinations of the water content and $D_{b}$ to the SRPC and SWRC.

Key words: organic carbon, soil bulk density, soil management, soil quality.

\section{INTRODUÇÃO}

A qualidade do solo em sistemas de manejo pode ser avaliada por indicadores simples (densidade do solo, resistência do solo à penetração e carbono orgânico do solo) ou por meio de funções de pedotransferências, definidas como funções (modelos de regressões) que transferem propriedades conhecidas do solo para propriedades do solo

IInstituto Agronômico do Paraná (IAPAR), CP 564, 87701-970, Paranavaí, PR, Brasil. E-mail: fidalski@iapar.br. Autor para correspondência.

"Departamento de Agronomia, Universidade Estadual de Maringá (UEM), Av. Colombo 5790, 87020-900, Maringá, PR, Brasil. Email: catormena@uem.br. 
desconhecidas, com as vantagens de maior facilidade e redução de custos (GREGORICH, 2006).

A implantação de pomares de laranjeira em solos com horizonte superficial arenoso derivados da Formação Caiuá, no noroeste do Paraná, a partir de 1988, coincidiu com a revisão mundial do sistema de manejo de solo em culturas perenes, baseado na manutenção da vegetação nas entrelinhas e no controle da vegetação nas linhas das plantas com herbicida (LIPECKI \& BERBEC, 1997). Transcorridas praticamente duas décadas de manejo de plantas permanentes nas entrelinhas de pomares de laranjeiras nessa região, persistem os questionamentos técnicos sobre as desvantagens do aumento da densidade do solo sob o rodado do trator de pneu, independentemente do aumento do teor de carbono orgânico do solo nas entrelinhas dos pomares (FIDALSKI \& STENZEL, 2006).

Por outro lado, observa-se que a avaliação do teor de água no solo e da resistência do solo à penetração tem sido realizada em condições específicas de umidade do solo, no momento da coleta de solo em pomares de laranjeiras (CINTRA et al., 1983; ABERCROMBIE \& PLESSIS, 1995), desconsiderando possíveis interações destas variáveis desde a saturação até o secamento da matriz do solo, em função da alteração da distribuição do diâmetro e da continuidade de poros drenados, resultante do tráfego de máquinas agrícolas ou da ação biológica das plantas. Esta alteração é possível de ser quantificada determinando-se simultaneamente o conteúdo de água no solo $(\theta)$ e a resistência do solo à penetração em diferentes potenciais mátricos ( $\Psi$ ) (SILVA \& KAY, 1997; TORMENA et al., 1998 e TORMENA \& SILVA, 2002), que resultam em curvas de retenção de água do solo e de resistência do solo à penetração. Estas curvas permitem a interpretação das relações entre a densidade do solo, os teores de carbono orgânico do solo, os teores de água e a resistência do solo à penetração em sistemas de manejo de solo.

Dada a característica arenosa da camada superficial dos solos da Formação Caiuá, a espécie e o manejo da cobertura permanente do solo pode aumentar o teor de carbono orgânico e a retenção de água no solo (RAWLS et al., 2003), com conseqüente redução da resistência do solo à penetração. Isto poderia eliminar a necessidade de revolvimento do solo para reduzir a resistência à penetração das raízes de citros e aumentar a disponibilidade de água no solo, preconizado em alguns estudos por ABERCROMBIE \& PLESSIS (1995) e SOUZA et al. (2004).

O objetivo deste estudo foi obter funções de pedotransferência (FPT) para as curvas de retenção de água e de resistência do solo à penetração, em sistemas de manejo com plantas de cobertura permanente nas entrelinhas de laranjeira "Pêra", em Argissolo Vermelho distrófico latossólico, no noroeste do Paraná.

\section{MATERIAL E MÉTODOS}

O estudo foi realizado em um experimento conduzido pelo Instituto Agronômico do Paraná (IAPAR), localizado na Fazenda São Judas Tadeu, município de Alto Paraná, no noroeste do Paraná (23ำ 5' S e $52^{\circ} 26^{\prime} \mathrm{W}$, $480 \mathrm{~m}$ de altitude). O relevo apresenta declividade média de $0,04 \mathrm{~m} \mathrm{~m}^{-1}$, perpendicular às linhas das plantas. O solo é um Argissolo Vermelho distrófico latossólico, textura areia, contendo 80, 25 e $895 \mathrm{~g} \mathrm{~kg}^{-1}$, respectivamente, de argila, silte e areia, na camada de 0-0,25m de profundidade (FIDALSKI et al., 2006).

O experimento foi implantado em 1993, com o plantio de laranjeira "Pêra” [Citrus sinensis (L.) Osb.] enxertada sobre o limoeiro "Cravo" (Citrus limonia Osb.). O delineamento experimental utilizado é de blocos ao acaso, dispostos perpendicularmente ao declive do terreno, com três repetições. Cada parcela experimental apresenta 15 plantas, com três linhas no espaçamento $7 \mathrm{~m}$, contendo cinco plantas espaçadas em $4 m$ dentro de cada linha.

Foram avaliados três tratamentos correspondendo aos sistemas de manejo implantados em 1993 nas entrelinhas do pomar: a) gramínea - grama mato-grosso ou batatais (Paspalum notatum Flügge); b) leguminosa - amendoim forrageiro (Arachis pintoi Krap. \& Greg.); c) vegetação espontânea - a vegetação espontânea foi controlada com o propósito de se manter baixa cobertura do solo, tendo sido utilizadas em 1998, 1999 e 2000, respectivamente, três, duas e uma aplicação de herbicida (Glyphosate). Anualmente, no período de novembro a março, roçada mecânica foi realizada para o controle da vegetação nas entrelinhas do tratamento gramínea.

A amostragem do solo foi realizada na primeira quinzena de maio de 2003, em três transeções perpendiculares aos troncos das três plantas centrais das duas entrelinhas das parcelas experimentais. Em cada uma das transeções foram identificados oito pontos de amostragem nas entrelinhas do pomar, sendo quatro pontos de amostragem no rodado (2,50 e 4,20m à esquerda e à direita do tronco da linha central das laranjeiras) e quatro pontos de amostragem no entrerrodado (3,20 e 3,50m à esquerda e à direita do tronco das laranjeiras). Nos 216 pontos de amostragem foram coletadas amostras indeformadas de solo na camada de 0,05-0,10m de profundidade, utilizando anéis 
metálicos com dimensões de 0,05m de diâmetro e de altura.

Amostras de solo agrupadas por tratamento (3), posição de amostragem (2) e repetição (3) foram saturadas por capilaridade dentro de bandejas de alumínio, por meio da manutenção de uma lâmina de água até $2 / 3$ da altura dos anéis, e, em seguida, submetidas a potenciais mátricos $(\Psi)$ de $-0,001,-0,002$, -0,004 -0,006 -0,008MPa em uma mesa de tensão, e $\Psi$ de $-0,01,-0,03,-0,05,-0,07,-0,1,-0,4,-1,5 \mathrm{MPa}$ por meio de pressões aplicadas em placas porosas (extratores de Richards). Após as amostras de solo atingirem equilíbrio hidráulico, foram pesadas para a determinação do teor de água $(\theta)$ e submetidas à determinação da resistência do solo à penetração nos respectivos $\Psi$. Foi feita uma determinação no centro das amostras, utilizando-se um penetrômetro estático similar ao descrito por TORMENA et al. (1998). Esse penetrômetro utiliza uma agulha com um cone na sua extremidade, o qual apresenta semi-ângulo de $30^{\circ}$ e área da base de $0,1256 \mathrm{~cm}^{2}$. A velocidade de penetração foi de $10 \mathrm{~mm}$ $\mathrm{min}^{-1}$, obtendo-se valores de resistência do solo à penetração a cada 0,6767 segundos, sendo utilizado o valor médio de 250 medidas obtidas na altura entre 1,00 a 4,69cm dos anéis metálicos, para representar a resistência do solo à penetração de cada amostra de solo. Em seguida, as amostras de solo foram pesadas e secadas em estufa a $\pm 110^{\circ} \mathrm{C}$ por 24 horas, para determinação do $\theta$ e da densidade do solo.

Após as determinações físicas, o solo contido nos anéis metálicos foi retirado, destorroado e tamisado em peneira de $2 \mathrm{~mm}$ de diâmetro de malha. Esse material de solo foi utilizado para a determinação do teor de carbono orgânico do solo pelo método Walkley \& Black, e da densidade de partículas do solo, conforme metodologias descritas pela EMBRAPA (1997).

Os dados originais da densidade do solo foram submetidos à análise exploratória para a identificação de possíveis valores atípicos (outliers), utilizando-se o critério [intervalo interquartil $\pm 1,5 \mathrm{x}$ (quartil inferior ou superior)]. Os dados médios da densidade do solo e do carbono orgânico do solo de cada parcela experimental e posição de amostragem foram submetidos às análises de variâncias por meio do modelo matemático de blocos ao acaso e ao teste Tukey de comparação de médias, com 5\% de probabilidade (SAS INSTITUTE, 2001).

A curva de retenção de água do solo foi ajustada pelo modelo matemático proposto por VAN GENUCHTEN (1980) com a restrição $(m=1-1 / n): \theta=\theta r$ $+\left\{(\theta \mathrm{s}-\theta \mathrm{r}) /\left[1+(\alpha \Psi)^{\mathrm{n}}\right]^{(1-1 / \mathrm{n})}\right\}$, em que $\Psi$ é o potencial mátrico (hPa); $\theta$ é o conteúdo de água $\left(\mathrm{m}^{3} \mathrm{~m}^{-3}\right)$; $\theta$ r é o conteúdo de água residual $\left(\mathrm{m}^{3} \mathrm{~m}^{-3}\right)$; $\theta$ s (conteúdo de água na saturação, $\left.\mathrm{m}^{3} \mathrm{~m}^{-3}\right)$; e a $\left(\mathrm{hPa}^{-1}\right)$ e $\mathrm{n}$ são coeficientes do modelo obtidos no ajuste dos dados. $\mathrm{O}$ valor de $\theta$ s foi obtido como: [ $\theta \mathrm{s}=$ (1-densidade do solo/2,62 $\left.\left.\mathrm{Mg} \mathrm{m}^{-3}\right)\right]$. O efeito das variáveis quantitativas (densidade do solo, carbono orgânico do solo e densidade de partículas do solo) e das variáveis qualitativas (tratamento e posição de amostragem) foi avaliado pela distribuição dos resíduos, conforme proposta por TORMENA \& SILVA(2002). Foi utilizada a variável indicadora para os tratamentos (NETER et al., 1996): gramínea (-1), vegetação espontânea (0) e leguminosa (1); e a variável Dummy para as posições de amostragem entrerrodado (0) e rodado (1). Os coeficientesde regressão do modelo matemático foram estimados pelo método de Gauss-Newton com o procedimento PROC NLIN (SAS INSTITUTE, 2001).

A relação funcional entre a resistência do solo à penetração, $\theta$ e a densidade do solo foi ajustada utilizando a função proposta por BUSSCHER (1990), a partir dos procedimentos descritos por TORMENA et al. (1998): [Resistência do solo à penetração $=\mathrm{c} \theta^{\mathrm{d}}$ (densidade do solo) $)^{\mathrm{e}}$, cuja solução foi obtida por meio da transformação logarítmica em uma equação linear múltipla: [ln (resistência do solo à penetração) $=\ln$ c + $\mathrm{d} \ln \theta+\mathrm{e} \ln$ (densidade do solo)], em que, $c, d$ e $e$ são os coeficientes de ajuste dos dados ao modelo matemático. As variáveis indicadoras e Dummy seguiram os mesmos valores utilizados nas curvas de retenção de água do solo.

A análise exploratória dos dados indicou a existência de um valor atípico (outlier), para o valor de densidade do solo de 1,44 $\mathrm{Mg} \mathrm{m}^{-3}$, no entrerrodado do tratamento gramínea, por enquadrar-se fora do limite interquartil inferior de $1,58 \mathrm{Mg} \mathrm{m}^{-3}$, razão pela qual este valor foi excluído do conjunto de dados para o ajuste das curvas de retenção de água do solo e de resistência do solo à penetração $(\mathrm{N}=71)$.

$\mathrm{O}$ ajuste dos dados da densidade de partículas do solo nas curvas de retenção de água do solo e de resistência do solo à penetração não foi significativo, razão pela qual foi utilizado o valor de $2,62 \mathrm{Mg} \mathrm{m}^{-3}$, correspondendo à média aritmética das 216 determinações realizadas nas próprias amostras de solo.

\section{RESULTADOS E DISCUSSÃO}

O valor médio da densidade do solo no rodado se diferenciou entre o tratamento gramínea e vegetação espontânea, mas não diferiu do tratamento leguminosa (Tabela 1). Os valores de densidade do solo foram superiores na posição entrerrodado para os 
Tabela 1 - Densidade e carbono orgânico do solo nos tratamentos (gramínea, leguminosa e vegetação espontânea) e posições de amostragem (rodado e entrerrodado)

\begin{tabular}{lcccc}
\hline Tratamento & $\begin{array}{c}\text { Densidade do } \\
\text { solo }\left(\mathrm{Mg} \mathrm{m}^{-3}\right)\end{array}$ & $\begin{array}{c}\text { Carbono orgânico } \\
\text { do solo }\left(\mathrm{g} \mathrm{kg}^{-1}\right)\end{array}$ \\
\hline \multicolumn{5}{c}{ Rodado } \\
Gramínea & 1,68 & $\mathrm{Ba}^{(1)}$ & 5,37 & $\mathrm{Aa}$ \\
Leguminosa & 1,70 & $\mathrm{ABa}$ & 5,02 & $\mathrm{Ab}$ \\
Vegetação espontânea & 1,72 & $\mathrm{Aa}$ & 4,63 & $\mathrm{Aa}$ \\
& \multicolumn{5}{c}{$\mathrm{Ab}$} & \\
Eramínea & 1,63 & $\mathrm{Ab}$ & \\
Leguminosa & 1,64 & $\mathrm{Ab}$ & 5,35 & $\mathrm{Aa}$ \\
Vegetação espontânea & 1,67 & $\mathrm{Ab}$ & 4,76 & $\mathrm{Aa}$ \\
\hline
\end{tabular}

(1) Médias seguidas pela mesma letra maiúscula e minúscula na coluna não diferem, respectivamente, entre os tratamentos dentro de cada posição de amostragem e entre posições de amostragem para o mesmo tratamento (Tukey, $\mathrm{P}<0,05$ ).

três tratamentos. Diferentemente, os teores de carbono orgânico do solo não se diferenciaram entre os tratamentos dentro de cada uma das posições de amostragem, mas observou-se, no tratamento leguminosa, menor teor de carbono orgânico do solo no rodado do que no entrerrodado. A maior compactação do solo no tratamento vegetação espontânea é atribuída à menor taxa de cobertura do solo, devido à dessecação da vegetação com herbicida (CINTRA et al., 1983). Os teores de água no solo para os diferentes potenciais mátricos variaram entre 0,05 e $0,38 \mathrm{~m}^{3} \mathrm{~m}^{-3}$, independentemente dos tratamentos, enquanto a resistência do solo à penetração apresentou amplitudes diferenciadas entre os tratamentos: gramínea (0,28-6,32MPa), leguminosa (0,27-10,36MPa) e vegetação espontânea (0,23-10,75MPa). O tratamento gramínea apresentou melhor qualidade física do solo para o desenvolvimento das raízes das plantas, expressa pelas variáveis densidade do solo e resistência do solo à penetração, mas não é possível explorar conjuntamente as relações com o carbono orgânico do solo e o conteúdo de água do solo. Estas poderiam ser explicadas pelas curvas de retenção de água do solo e de resistência do solo à penetração, as quais são dependentes dos potenciais mátricos $(\Psi)$ aplicados.

Os coeficientes da curva de retenção de água do solo da função de VAN GENUCHTEN (1980), ajustada aos dados de $\theta(\Psi)$, explicaram 94\% $(\mathrm{P}<0,0001)$ da variabilidade do $\theta$. Houve efeito dos tratamentos no coeficiente $\theta$ r e da densidade do solo no coeficiente n, indicando que o manejo da cobertura do solo influência o $\theta$ com o secamento do solo e o aumento da compactação reduz a curvatura da curva de retenção de água do solo, reduzindo a amplitude de água retida pelo solo em diferentes $\Psi$. Estes coeficientes foram estatisticamente significativos, por terem apresentado intervalos de confiança sem nenhum valor nulo (Tabela 2). Não foi constatado efeito significativo das posições de amostragem para os coeficientes da curva de retenção de água do solo, fato também relatado por TORMENA et al. (1998). A independência entre as curvas de retenção de água do solo para as posições de amostragem se deve à elevada freqüência relativa de amostras de solo que apresentaram a mesma amplitude de valores de densidade do solo no rodado e no entrerrodado, 85, 75 e 83\%, respectivamente, para os tratamentos gramínea, leguminosa e vegetação espontânea. Os sinais dos coeficientes $\left(a_{1}\right.$ e $\left.a_{2}\right)$ da curva de retenção de água do solo indicam simultaneamente efeitos positivo e negativo, respectivamente, para os teores de carbono orgânico do solo e o tratamento no valor de $\theta$ r (Tabela 2). De acordo com RAWLS et al. (2003), para solo com baixo teor de argila, um pequeno aumento de carbono orgânico do solo resulta no aumento de $\theta$ r.

O ajuste dos dados da resistência do solo à penetração em função de $\theta$ e da densidade do solo explicaram 87\% $(\mathrm{P}<0,0001)$ da variabilidade da resistência do solo à penetração (Tabela 2). Os coeficientes de regressão da curva de resistência do solo à penetração indicam que esta varia negativamente com $\theta$ (coeficiente $d$ ) e positivamente com a densidade do solo (coeficiente $e$ ). O efeito positivo dos teores de carbono orgânico do solo no intercepto do modelo da curva de resistência do solo à penetração é atribuído à maior estabilidade das ligações entre as partículas primárias do solo (SILVA \& KAY, 1997). No tratamento gramínea, as relações entre o carbono orgânico do solo, a menor compactação (Tabela 1) e o aumento de retenção de água do solo (Tabela 2 e Figura 1a), estão associados no menor incremento da resistência do solo à penetração em função da densidade do solo conferido pelo menor valor do coeficiente $e$ da curva de resistência do solo à penetração (Tabela 2 e figura 1b). A identificação da capacidade da gramínea em mitigar as restrições físicas e hídricas deste solo nas entrelinhas do pomar de citros possibilita a substituição da intervenção mecânica com subsolagem proposta por ABERCROMBIE \& PLESSIS (1995).

$\mathrm{O}$ fato de ter sido ajustado simultaneamente nas funções de pedotransferência das curvas de retenção de água e resistência do solo à penetração (Tabela 2), o efeito do teor de carbono orgânico do solo, da densidade do solo e do tratamento é atribuído à consolidação dos sistemas de manejo de solo nas entrelinhas durante dez anos (FIDALSKI et al., 2006). 
Tabela 2 - Coeficientes, valores estimados, intervalos de confiança e probabilidades das funções de pedotransferência para as curvas de retenção da água do solo ajustadas pelo modelo Van Genuchten e de resistência do solo à penetração, com as variáveis independentes teor de carbono orgânico do solo, densidade do solo e tratamento.

\begin{tabular}{|c|c|c|c|}
\hline Coeficiente & Valor & Intervalo de confiança & Probabilidade \\
\hline \multicolumn{4}{|c|}{ Curva de retenção de água do solo $\left(\mathrm{R}^{2}=0,94 ; \mathrm{P}<0,0001\right): \theta=\theta \mathrm{r}+\left\{(\theta \mathrm{s}-\theta \mathrm{r}) /\left[1+(\alpha \Psi)^{\mathrm{n}}\right]^{(1-1 / \mathrm{n})}\right\}$} \\
\hline$\alpha$ & 0,0192 & 0,0178 a 0,0205 & \\
\hline$\theta \mathrm{r}$ & \multicolumn{3}{|c|}{$\left[a_{0}+\left(a_{1} \times\right.\right.$ carbono orgânico do solo $)+\left(a_{2} \times\right.$ tratamento $\left.)\right]$} \\
\hline$a_{0}$ & 0,0531 & 0,0290 a 0,0772 & \\
\hline$a_{1}$ & 0,0062 & 0,0021 a 0,0103 & \\
\hline$a_{2}$ & $-0,0090$ & $-0,0147$ a $-0,0032$ & \\
\hline $\mathrm{n}$ & \multicolumn{3}{|c|}{$\left[n_{0}+\left(n_{1} \times\right.\right.$ densidade do solo $\left.)\right]$} \\
\hline$n_{0}$ & 10,4807 & 6,0376 a 14,9238 & \\
\hline$n_{1}$ & $-4,7405$ & $-7,3357$ a $-2,1453$ & \\
\hline \multicolumn{4}{|c|}{ Curva de resistência do solo à penetração $\left(\mathrm{R}^{2}=0,87 ; \mathrm{P}<0,0001\right): \ln (\mathrm{RP})=\mathrm{c}+\mathrm{d}(\ln \theta)+\mathrm{e}(\ln$ densidade do solo $)$} \\
\hline $\mathrm{c}$ & \multicolumn{3}{|c|}{$\left[c_{0}+\left(c_{1} \times\right.\right.$ tratamento $)+\left(c_{2} \times\right.$ carbono orgânico do solo $\left.)\right]$} \\
\hline$c_{0}$ & $-5,0032$ & & $<0,0001$ \\
\hline$c_{1}$ & $-1,0664$ & & 0,0200 \\
\hline$c_{2}$ & 0,0632 & & 0,0009 \\
\hline$d$ & $-1,1211$ & & $<0,0001$ \\
\hline e & \multicolumn{3}{|c|}{$\left[e_{0}+\left(e_{1} \times\right.\right.$ tratamento $\left.)\right]$} \\
\hline$e_{0}$ & 5,8062 & & 0,0039 \\
\hline$e_{1}$ & 1,9031 & & 0,0345 \\
\hline
\end{tabular}

$\theta$ (conteúdo de água volumétrica, $\left.\mathrm{m}^{3} \mathrm{~m}^{-3}\right) ; \Psi$ (Potencial mátrico, $\mathrm{hPa}$ ); ln (logaritmo natural na base e $\left.=2,7183\right)$ e tratamento $($ variável indicadora): gramínea (-1), vegetação espontânea (0) e leguminosa (1); RP (resistência do solo à penetração).

Alternativamente, a partir das funções de pedotransferência, dos valores originais de densidade do solo, do carbono orgânico do solo, de $\theta$, da resistência do solo à penetração e das variáveis indicadoras dos tratamentos (Tabela 2), foram obtidas funções de pedotransferência matematicamente mais simples para as curvas de retenção de água do solo (Figura 1a) e de resistência do solo à penetração (Figura 1b), dependentes apenas da determinação da densidade do solo e de $\theta$.

A manutenção da leguminosa nas entrelinhas aumentou os riscos de deficiência hídrica às plantas do pomar, indicado pela curva de retenção de água do solo (Figura 1a) e confirmados pelas variáveis fisiológicas das laranjeiras (taxa de fotossíntese, condutância estomática e potencial da água nas folhas de laranjeiras) e hídricas (teor de água no solo), monitoradas nestes tratamentos por FIDALSKI et al. (2006). Os resultados do presente estudo reiteram as recomendações do manejo nas entrelinhas dos pomares vegetados com gramínea, conforme proposto por LIPECKI \& BERBEC (1997). A melhor qualidade física e hídrica do solo nas entrelinhas vegetadas permanentemente com gramínea foi confirmada neste experimento pelas melhores relações hídricas e fisiológicas das laranjeiras, o que sustentou a produção de frutos das laranjeiras "Pêra" em relação aos tratamentos leguminosa e vegetação espontânea (FIDALSKI et al., 2006).

\section{CONCLUSÕES}

As funções de pedotransferência foram dependentes da densidade do solo, do carbono orgânico e do tratamento para o ajuste das curvas de retenção de água $\left(\mathrm{R}^{2}=0,94\right)$ e de resistência do solo à penetração $\left(R^{2}=0,87\right)$. O manejo da vegetação espontânea com herbicida aumenta a compactação do solo, reduz a retenção de água e aumenta a resistência do solo à penetração. A leguminosa reduz a retenção de água do solo e aumenta a resistência do solo à penetração, e a gramínea melhora a qualidade física e

Ciência Rural, v.37, n.5, set-out, 2007. 


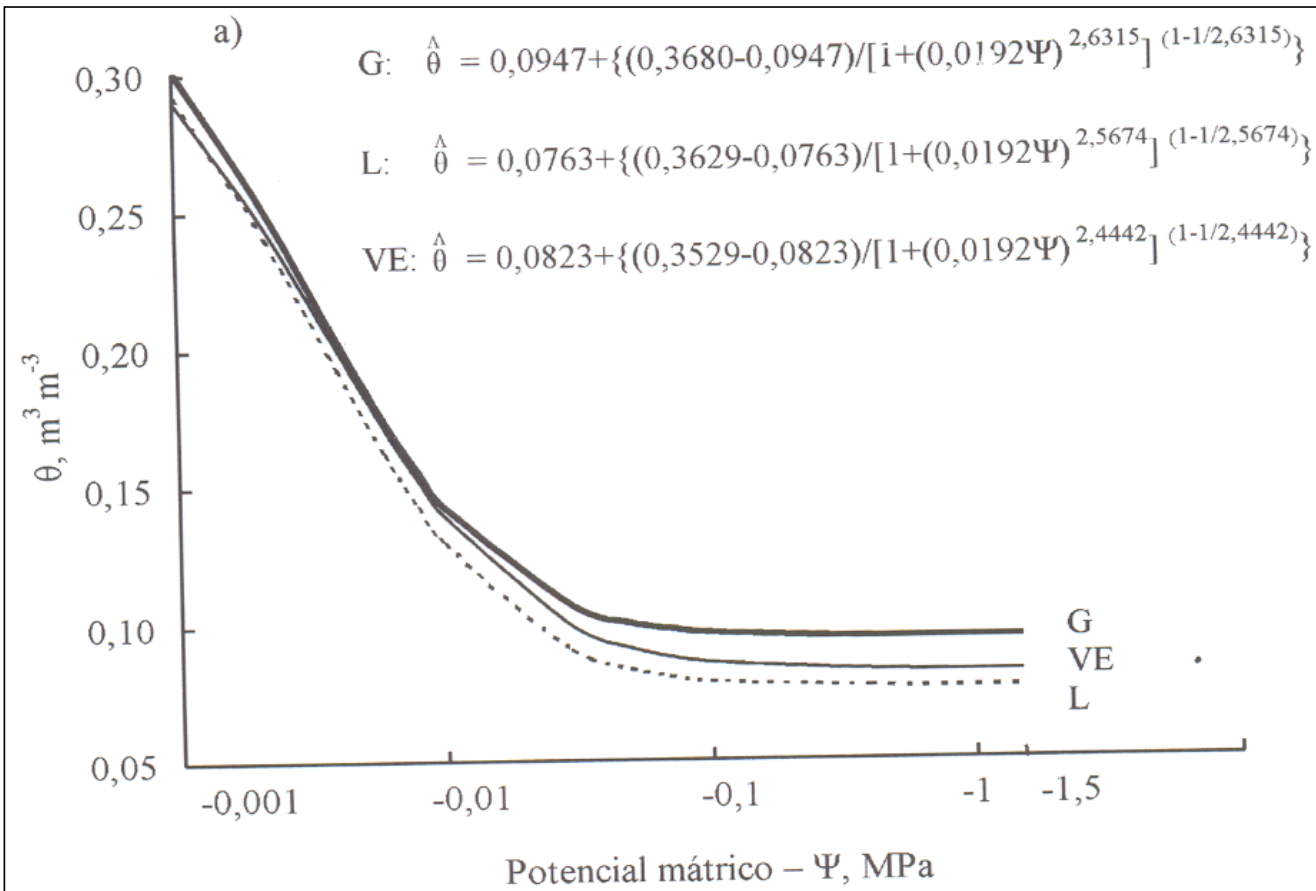

b)

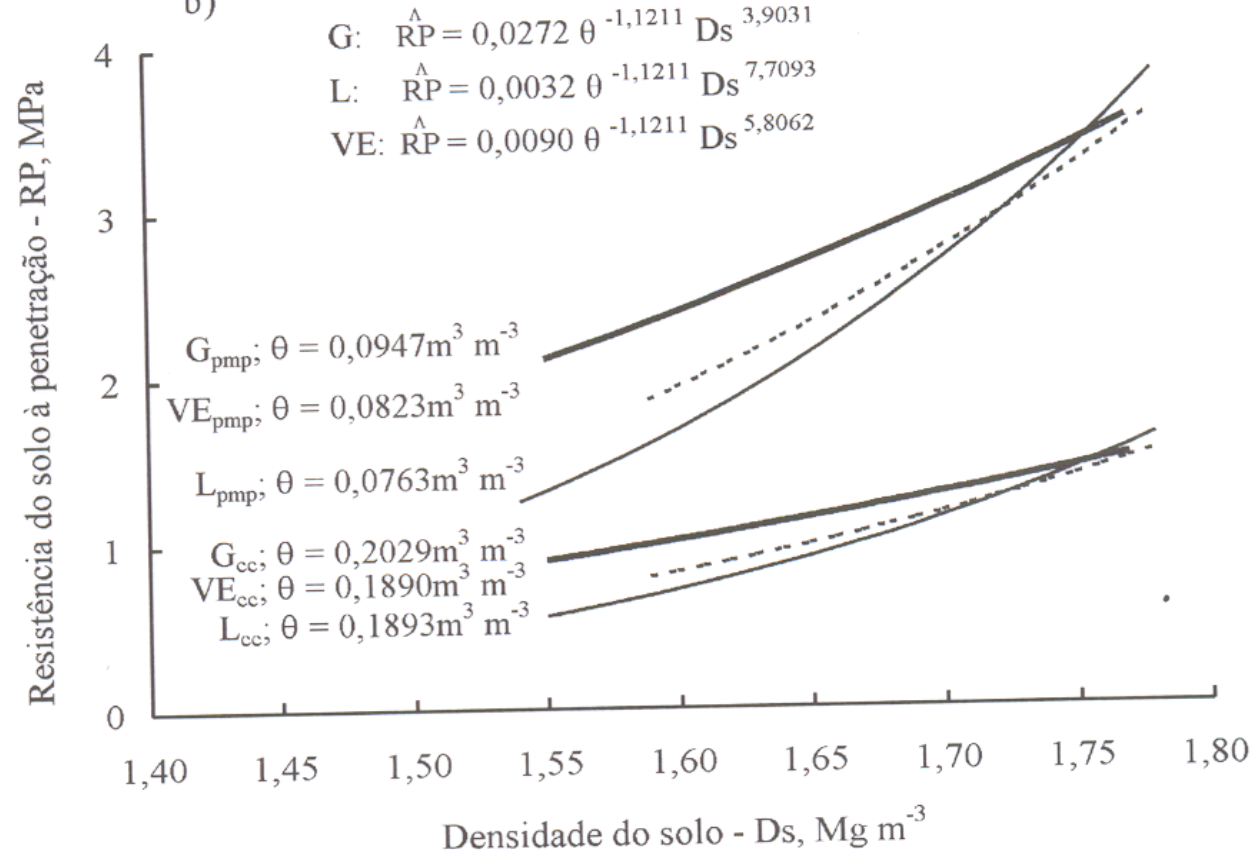

Figura 1 - Funções de pedotransferência simplificadas das curvas de retenção de água do solo ajustadas pelo modelo Van Genuchten (a); e das curvas de resistência do solo à penetração ajustadas pelo modelo não linear, correspondentes aos teores de água nos potenciais mátricos da capacidade de campo (cc=-0,008MPa) e do ponto de murcha permanente (pmp=-1,5MPa) (b), para os tratamentos gramínea (G), leguminosa (L) e vegetação espontânea (VE). 
hídrica do solo nas entrelinhas. Alternativamente, as funções de pedotransferência exigem apenas as determinações dos teores de água e da densidade do solo para as curvas de retenção de água e de resistência do solo à penetração.

\section{REFERÊNCIAS}

ABERCROMBIE, R.A.; PLESSIS, S.F.D. The effect of alleviating soil compaction on yield and fruit size in an established navel orange orchard. Journal of the Southern African Socociety for Horticulture Sciences, Pietermaritzburg, v.5, n.2, p.85-89, 1995.

BUSSCHER, W.J. Adjustment of flat-tipped penetrometer resistance data to a common water content. Transactions of the ASAE, Saint Joseph, v.33, n.2, p.519-524, 1990.

CINTRA, F.L.D. et al. Caracterização física do solo submetido a práticas de manejo em pomar de laranja Baianinha. Pesquisa Agropecuária Brasileira, Brasília, v.18, n.2, p.173-179, 1983.

EMBRAPA. Manual de métodos de análises de solos. Rio de Janeiro: Serviço Nacional de Levantamento e Conservação de Solos, 1997. 212p.

FIDALSKI, J.; STENZEL, N.M.C. Nutrição e produção da laranjeira "Folha Murcha" em porta-enxertos e plantas de cobertura permanente na entrelinha. Ciência Rural, Santa Maria, v.36, n.3. p.807-813, 2006.

FIDALSKI, J. et al. Produção de laranja com plantas de cobertura permanente na entrelinha. Pesquisa Agropecuária Brasileira, Brasília, v.41, n.6, p.927-935, 2006.
GREGORICH, G. Quality. Encyclopedia of Soil Science. Columbus: Taylor \& Francis Group, 2006. p.1388-1391.

LIPECKI, J.; BERBEC, S. Soil management in perennial crops: orchards and hop gardens. Soil Tillage and Research, Amsterdam, v.43, n.1/2, p.169-184, 1997.

NETER, J. et al. Applied linear statistical models. 4.ed. Chicago: IRWIN, 1996. 1408p..

RAWLS, W.J. et al. Effect of soil organic carbon on soil water retention. Geoderma, Amsterdam, v.116, n.1, p.61-76, 2003.

SAS INSTITUTE. SAS/STAT user's guide. Version 8.2. Cary, 2001. 943p.

SILVA, A.P.; KAY, B.D. Estimating the least limiting water range of soil from properties and management. Soil Science Society of America Journal, Madison, v.61, n.3, p.877883, 1997.

SOUZA, L.D. et al. Disponibilidade de água em pomar de citros submetido a poda e subsolagem em Latossolo Amarelo dos Tabuleiros Costeiros. Revista Brasileira de Fruticultura, Jaboticabal, v.26, n.1, p.69-73, 2004.

TORMENA, C.A. et al. Caracterização do intervalo hídrico ótimo de um Latossolo Roxo sob plantio direto. Revista Brasileira de Ciência do Solo, Viçosa, v.22, n.4, p.573581, 1998.

TORMENA, C.A.; SILVA, A.P. Incorporação da densidade no ajuste de dois modelos à curva de retenção de água no solo. Revista Brasileira de Ciência do Solo, Viçosa, v.26, n.2, p.305-314, 2002.

VAN GENUCHTEN, M.T. A closed-form equation for predicting the hydraulic conductivity of unsaturated soils. Soil Science Society of America Journal, Madison, v.44, n.5, p.892-898, 1980. 